\title{
Os grupos funcionais fitoplanctônicos nos reservatórios do Sistema Cantareira, São Paulo, Brasil'
}

\author{
Larissa Gonçalves Santos ${ }^{1,2}$, Leila dos Santos Machado², Viviane Moschini-Carlos² \\ \& Marcelo Pompêo ${ }^{3}$
}

\begin{abstract}
'Parte de dissertação de mestrado da primeira autora no Programa de Pós-Graduação em Ciências Ambientais da Universidade Estadual Paulista, São Paulo.

2Universidade Estadual Paulista "Júlio de Mesquita Filho", Instituto de Ciências e Tecnologia, Campus Experimental de Sorocaba Av. Três de Março, 51 1, Alto da Boa Vista, CEP 18087-180, Sorocaba, São Paulo, Brasil. laribtos@gmail.com

3Universidade de São Paulo, Instituto de Biociência, Departamento de Ecologia, Rua do Matão 321, Travessa 14, Cidade Universitária, CEP 05508-090, São Paulo, Brasil.
\end{abstract}

RESUMO - Este estudo objetivou verificar se a comunidade fitoplanctônica responde às diferentes trofias nos reservatórios do Sistema Cantareira, baseando-se na distribuição dos grupos funcionais fitoplanctônicos (GFF). As amostragens foram realizadas nos reservatórios Jaguari-Jacareí, Cachoeira, Atibainha e Paiva Castro, nos períodos de maio/junho e novembro/dezembro de 2013. Em geral, a maioria dos reservatórios apresentou condições mesotróficas, sendo o Jaguari, o que apresentou maior trofia, supereutrófico. Nós encontramos variação espaço-sazonal da trofia do reservatório a montante, com registros de elevada biomassa do GFF Lo na primeira amostragem e na segunda amostragem elevada biomassa de cianobactérias filamentosas do GFF Sn, comprovando a existência de correspondência entre descritores ambientais e a variação da biomassa dos GFF. A classificação trófica destes ambientes baseada em GFF foi eficaz, pois a ocorrência dos GFF tolerantes ou sensíveis foi compatível com as características ambientais observadas.

Palavras-chave: bioindicadores, biomassa, distribuição espacial, eutrofização

\begin{abstract}
The phytoplankton functional groups in the reservoirs of the Cantareira System, São Paulo, Brazil. This study aimed to verify if the phytoplankton community responds to different trophic states in the reservoirs of the Cantareira System, based on the distribution of phytoplankton functional groups (GFF). The samplings were carried out in the Jaguari-Jacareí, Cachoeira, Atibainha and Paiva Castro reservoirs, in the periods of May/June and November/December of 2013. Overall, most of the reservoirs were mesotrophic, where Jaguari, had the highest trophic state, classified as supereutrophic. We found a spatiotemporal variation of trophy of the upstream reservoir trophy, with high GFF biomass records in the first sampling and in the second, high biomass of GFF filamentous cyanobacteria, showing a correlation between environmental variables and the biomass variation of the GFF. The trophic classification of these environments based on GFF was effective, since the occurrence of tolerant or sensitive GFF was compatible with the environmental characteristics.
\end{abstract}

Keywords: bioindicators, biomass, spatial distribution, eutrophication

\section{INTRODUÇÃO}

Os reservatórios são ambientes ecologicamente complexos e heterogêneos, sujeitos a impactos ocasionados pelo aumento das atividades antrópicas em seu entorno (Santos 2014), como por exemplo, lançamento de esgotos domésticos, compostos tóxicos procedentes de pesticidas e resíduos químicos das atividades industriais (SerafimJúnior et al. 2010), podendo levar ao enriquecimento excessivo de nutrientes nesses ambientes, em especial de fósforo e nitrogênio, produzindo o efeito conhecido como eutrofização (Pinto-Coelho et al. 1999).

O sudeste brasileiro, especificamente a Região Metropolitana de São Paulo (RMSP), possui oito sistemas de abastecimento de água: Cantareira, Guarapiranga, Rio Claro, Alto Tietê, Rio Grande, Alto Cotia, Baixo Cotia e
Ribeirão da Estiva. O Sistema Cantareira é um dos sistemas mais importantes do Estado de São Paulo, o qual, fornece água para cerca de metade da população $(9$ milhões de pessoas) residente na RMSP (Whately \& Cunha 2007) e, suas águas possuem melhor qualidade, quando comparadas às outras massas de água empregadas no abastecimento público dessa mesma região (Cardoso-Silva et al. 2014). Contudo, devido aos usos múltiplos de suas águas, houve uma piora na qualidade destas, imposta ao crescimento desordenado no entorno dos reservatórios e, despejo de material orgânico observado, principalmente, no Paiva Castro (Giatti 2000).

O reservatório Paiva Castro apresenta sinais de comprometimento na qualidade das águas provenientes da represa Atibainha, devido às aplicações de sulfato de cobre na saída de água do túnel, no leito do rio Juqueri, 
para o controle de proliferação de algas e plantas aquáticas (Otomo et al. 2015). O aumento na quantidade de nitrogênio e fósforo lançados no Sistema pode ser considerado o fator desencadeador desse crescimento algal, potencializando os efeitos da eutrofização artificial. Dessa forma, a qualidade da água de qualquer um dos reservatórios à montante afeta a qualidade da água que chega ao último reservatório do Sistema, o Paiva Castro (Pômpeo et al. 2017).

Estes fatores são problemas cada vez mais comuns nos reservatórios brasileiros destinados ao abastecimento público, intensificando a degradação ambiental e trazendo como consequências a diminuição da disponibilidade de água e o comprometimento da qualidade dos corpos hídricos (Buzelli \& Cunha-Santino 2013).

Uma maneira de se reconhecer o nível de degradação e qualificar o estado trófico de um corpo hídrico é através do monitoramento físico, químico e biológico da água. Quanto ao estudo das características biológicas da água, as análises por meio da comunidade fitoplanctônica têm contribuído cada vez mais no monitoramento dos ecossistemas aquáticos, devido à elevada sensibilidade desses organismos em relação às variações das condições ambientais (Brasil \& Huszar 2011). Devido à esta sensibilidade, estes organismos podem ser utilizados como bioindicadores da qualidade da água, já que sua composição e distribuição podem variar de acordo com a trofia do ambiente.

Dessa forma, visando compreender a estruturação e funcionalidade da comunidade fitoplanctônica em diferentes condições ambientais, foi proposto agrupar esses organismos em categorias (Reynolds et al. 2002). Estes autores utilizaram-se de fatores ambientais para estabelecer uma classificação funcional apropriada para determinados grupos fitoplanctônicos e seu nicho ecológico, e agruparam diferentes espécies em trinta e um grupos funcionais (Nishimura et al. 2015). Entretanto, Padisák et al. (2009), revisaram este trabalho e reagruparam as espécies fitoplanctônicas em quarenta grupos funcionais (GF), designados por códigos ou códons alfanuméricos, com base em suas estratégias de sobrevivência, intolerâncias e sensibilidades.

Brasil \& Huszar (2011), estudaram a relação entre os grupos funcionais propostos por Reynolds et al. (2002) e os diferentes tipos de sistemas aquáticos brasileiros, como por exemplo, estuários, rios, lagoas costeiras, lagos de inundação, lago natural profundo e grandes reservatórios, em todas as regiões do país, e chegaram a uma conclusão que classificar os organismos em grupos funcionais é uma ótima ferramenta para sintetizar os tipos de comunidades em ecossistemas aquáticos brasileiros, levando-se em consideração o tipo de ambiente e seu estado trófico.

Neste sentido, o estudo comparativo de reservatórios com diferentes espectros tróficos é uma forma de identificar padrões e regras gerais que expliquem, pelo menos parcialmente, as associações fitoplanctônicas ao longo de determinada condição trófica; e conhecer a comunidade fitoplanctônica em ecossistemas tão importantes como são os reservatórios de abastecimento, contribuindo para uma abordagem mais holística quanto ao aspecto ecológico destes ambientes (Nishimura 2008).
Portanto, visando obter informações sobre as características ambientais do Sistema Cantareira durante o período atípico de estiagem abordado pelo presente estudo; objetivou-se verificar se a comunidade fitoplanctônica responde às diferentes trofias nos reservatórios do Sistema Cantareira, a partir de uma análise baseada nos grupos funcionais fitoplanctônicos.

\section{MATERIAL E MÉTODOS}

\section{Área de estudo}

O Sistema Cantareira está localizado na região norte do Estado de São Paulo, ocupando uma área de aproximadamente $8.051 \mathrm{~km}^{2}$ e abrangendo as bacias hidrográficas do alto rio Tietê e do Piracicaba. É considerado um dos principais sistemas de abastecimento de água composto por seis reservatórios ligados entre si, por túneis ao norte da Região Metropolitana de São Paulo (RMSP), sendo eles: (1) reservatório Jaguari e (2) reservatório Jacareí, ambos na região de Bragança Paulista; (3) reservatório Cachoeira, no município de Piracaia; (4) Atibainha, em Nazaré Paulista; (5) Paiva Castro, em Franco da Rocha e (6) reservatório Águas Claras, em Caieiras (Whately \& Cunha 2007) (Fig. 1). O clima da região é mesotérmico subtropical com inverno frio e seco (de abril a setembro) e verão quente e chuvoso (outubro a março) (Barros 2012), com temperaturas médias anuais que vão desde 18 a $22^{\circ} \mathrm{C}$, e precipitação média anual de $1400 \mathrm{~mm}$ (Moraes et al. 1998).

\section{Metodologia de coleta e análise da água}

As coletas foram realizadas nos meses de maio/junho e novembro/dezembro de 2013, em estações localizadas nas zonas próximas aos rios principais, regiões da barragem e nas regiões de influência das transferências hídricas, em cinco reservatórios do Sistema Cantareira (Jaguari-Jacareí, Cachoeira, Atibainha e Paiva Castro).

Para a caracterização da massa de água e de seu estado trófico, em cada estação de coleta foram realizadas medidas em perfil de temperatura $(\mathrm{T})$, oxigênio dissolvido (OD), $\mathrm{pH}$ e condutividade elétrica (CE) da água (sonda multiparâmetros YSI 556 MPS).

Os dados da pluviometria foram obtidos por meio do site da Companhia de Saneamento e Abastecimento de Água do Estado de São Paulo (SABESP 2014). Com a água coletada foram determinadas as concentrações das variáveis ambientais: transparência (Disco de Secchi), nitrito e nitrato (Mackereth et al. 1978); amônio (Koroleff 1976); fósforo total (Valderrama 1981); clorofila-a e feofitina (Lorenzen 1967); sólidos totais em suspensão (Wetzel \& Likens 1991).

\section{Metodologia de Coleta e Análise do fitoplâncton}

Para a análise qualitativa das amostras de fitoplâncton utilizou-se rede de malha de $20 \mu \mathrm{m}$ para realizar o arraste horizontal na superfície da coluna d'água e por fim, as 

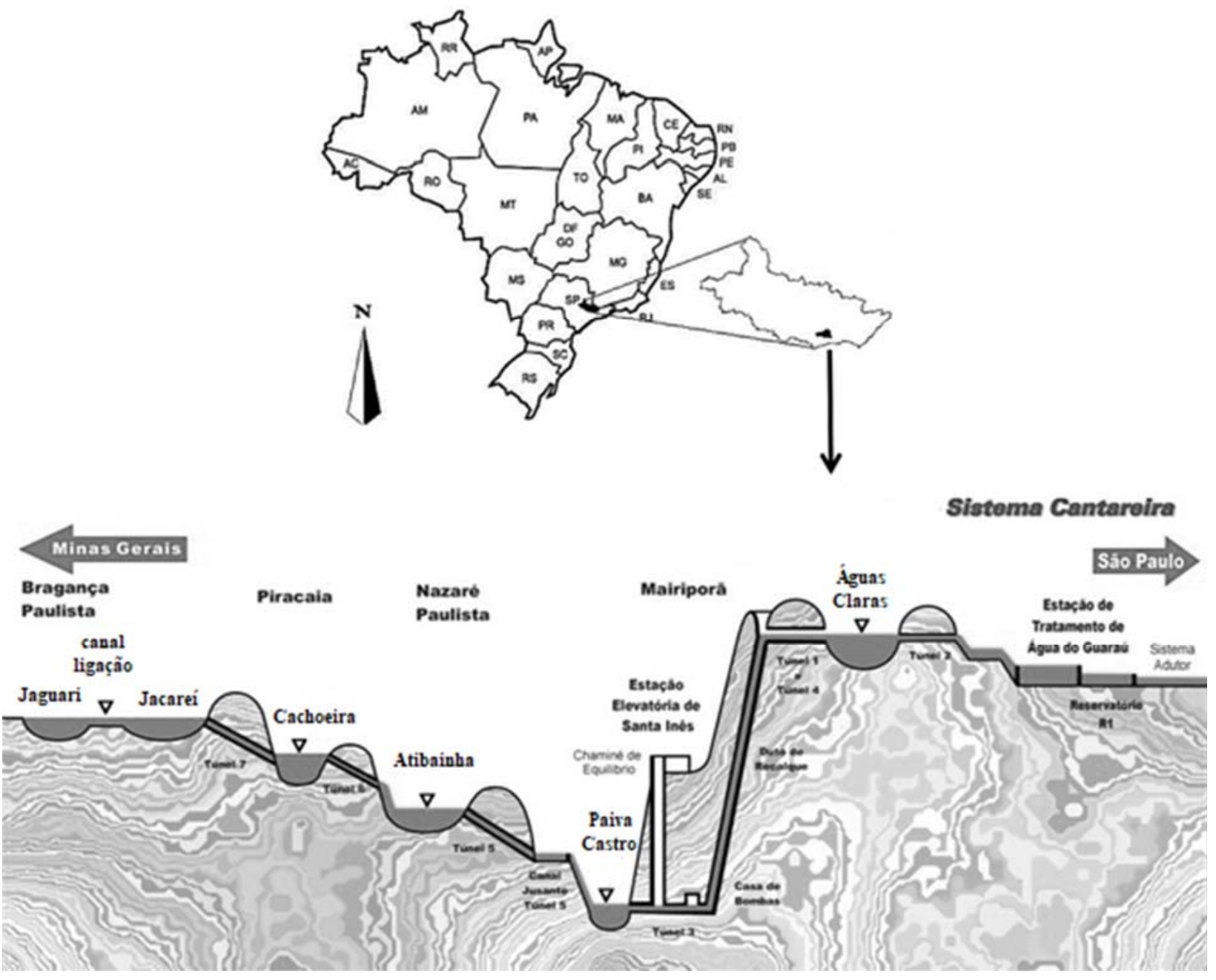

Fig. 1. Região Metropolitana de São Paulo, Brasil. Esquema representativo dos reservatórios do Sistema Cantareira. Fonte: Modificado Agência Nacional de Águas (2015).

amostras foram acondicionadas em frascos de $100 \mathrm{ml} \mathrm{e}$ fixadas com formaldeído a $4 \%$.

Além desta amostragem descrita acima, também foi realizado coletas de amostras integradas de água superficial de acordo com a metodologia (INAG 2009). Em cada local de amostragem utilizou-se de uma mangueira plástica de jardim de uma polegada de diâmetro (Navarro et al. 2006; Becker et al. 2010; Pômpeo et al. 2017), com modificações. Essas mangueiras eram de 5, 3 e $2 \mathrm{~m}$ de comprimento, e foram usadas quando as profundidades dos locais de amostragem eram maiores que $10 \mathrm{~m}$, entre 10 e $5 \mathrm{~m}$, e menores que 5 $\mathrm{m}$, respectivamente. A mangueira foi repetidamente aberta até que um volume de 5 litros de água fosse coletado, e em seguida foi armazenado em uma garrafa de plástico e mantido dentro de uma bolsa fria até ser transportado para o laboratório. Uma alíquota de $100 \mathrm{ml}$ desta amostra foi retirada e armazenada em frascos de vidro cobertos por papel alumínio, utilizando lugol acético $1 \%$ como fixador, para posterior análise quantitativa do fitoplâncton.

A identificação da composição fitoplanctônica foi realizada até o menor nível taxonômico possível, utilizandose chaves de classificação disponíveis nas literaturas impressas, como por exemplo, Round $(1965,1971)$ para Chlorophyceae, Chrysophyceae e Euglenophyceae; Anagnostidis \& Komarék $(1988,1990)$ e Komarék \& Anagnostidis $(1986,2005)$ para Cyanophyceae; Krammer \& Lange-Bertalot. (1986), Torgan et al. (1999), Bicudo \& Menezes (2006), Nascimento (2012), para Bacillariophyceae.

Em laboratório a contagem dos organismos fitoplanctônicos foi feita em microscópio invertido Zeiss, modelo Axiovert 40C, no aumento de 400 vezes, com o objetivo de contar 60 ou mais campos em transectos (Uhelinger 1964) e quantificar, pelo menos, 100 organismos da espécie mais abundante (Lund et al. 1958).

A densidade foi estimada de acordo com o método de Utermöhl (1958) e o biovolume das algas foi calculado multiplicando-se a densidade de cada espécie pelo volume médio de suas células ou colônias (Torgan 1997). Os volumes dos indivíduos foram calculados com base em formas geométricas apresentadas por Hillenbrand et al. (1999) e Sun \& Liu (2003). As espécies que contribuíram com mais de $5 \%$ para a biomassa total do fitoplâncton foram classificadas em grupos funcionais, utilizando-se os critérios de Reynolds et al. (2002) revisto por Padisák et al. (2009). 


\section{Tratamento dos dados}

A ordenação das unidades amostrais no tempo e no espaço foi realizada através da análise de correspondência canônica - ACC (Ter Braak 1986), a partir de dados biológicos - biomassa dos grupos funcionais fitoplanctônicos e dados ambientais referentes aos dois períodos de coleta estudados.

Os dados das variáveis abióticas foram padronizados aplicando o método de score $\mathrm{Z}$ e os dados bióticos para $\log (\mathrm{x}+1)$, foram realizadas análises de correlação linear de Pearson $(p \leq 0,05)$ dos dados abióticos para eliminação do efeito de colinearidade.

Durante o período de maio/junho de 2013, as variáveis ambientais selecionadas de acordo com a correlação linear de Pearson foram: pH; NID (nitrogênio inorgânico dissolvido); PT (fósforo total); Chla (clorofila-a); SS (sólidos em suspensão); \%SIS (frações de sólidos inorgânicos em suspenção); CE (condutividade elétrica) e Zeu (zona eufótica) e no período de novembro/dezembro de 2013, as variáveis ambientais selecionadas foram: Zeu, NID, PT, Chla, SS, \%SOS (frações de sólidos orgânicos em suspensão), OD (oxigênio dissolvido) e T (temperatura).

$\mathrm{O}$ índice do estado trófico (IET) foi calculado com base na média aritmética dos dados de fósforo total e clorofila a, conforme Carlson (1977) e modificado por Lamparelli (2004) para reservatórios. As análises estatísticas foram efetuadas por meio do software o Programa Past Paleontological Statistics (Hammer et al. 2001) e R Project for Statistical Computing ${ }^{\circledR}$.

\section{RESULTADOS E DISCUSSÃO}

Durante o período de estudo (maio/junho e novembro/ dezembro de 2013), a partir do índice pluviométrico que abrangeu o Sistema Cantareira, foi constatada precipitação máxima no mês de fevereiro com $249,2 \mathrm{~mm}$, ficando acima da média histórica que foi de $206,5 \mathrm{~mm}$, e no mês de agosto a precipitação foi a menor, apresentando 7,1 $\mathrm{mm}$. Contudo, nos meses de coleta, a precipitação ficou abaixo da média histórica, apresentando um período atípico de estiagem, não caracterizando ao certo os períodos de seca e chuva.
Entretanto, de acordo com Tavares et al. (2015), comportamentos climáticos atípicos têm sido cada vez mais presenciados na região Sudeste do Brasil, normalmente no final do mês de maio e início de junho, ocorreu uma redução das chuvas e da umidade do ar, caracterizando esses meses como secos, já no final do mês de novembro para início do mês de dezembro ocorreu o final da seca e o início da chuva, caracterizando esses meses como chuvosos (Fig. 2).

O período tradicionalmente chuvoso na região sudeste, sofreu com uma estiagem atípica, os índices pluviométricos observados não foi suficiente para repor a água consumida diariamente no Sistema Cantareira.

\section{Variáveis físicas, químicas e biológicas}

Na Figura 3 é possível visualizar como as variáveis se comportam de acordo com os dois períodos de amostragem.

Dentre os cincos reservatórios do Sistema Cantareira nos quais as coletas foram realizadas, no reservatório Jaguari foi registrado a maior trofia em relação aos demais, corroborando os resultados apresentados por De-Carli et al. (2017). Neste reservatório, as maiores concentrações de nutrientes foram observadas principalmente nos meses de novembro/dezembro, com altas concentrações da série nitrogenada (NID=900 $\mu \mathrm{g} / \mathrm{L}$; NH4 $=117,9 \mu \mathrm{g} / \mathrm{L}$; $\mathrm{NO} 3=713,4 \mu \mathrm{g} / \mathrm{L}$ ), clorofila-a $14,1 \mu \mathrm{g} / \mathrm{L}$, sólidos em suspensão $27,4 \mathrm{mg} / \mathrm{L}$, e apenas o fósforo, no período (maio/junho) ficou acima do estabelecido pelo CONAMA $357 / 05$, registrando $47,3 \mu \mathrm{g} / \mathrm{L}$, corroborando os resultados apresentados por Pômpeo et al. (2017).

Com base nos valores de $\mathrm{pH}$, pôde-se caracterizar o ambiente como ácido no primeiro período de amostragem (maio/junho) e alcalino no segundo período (novembro/ dezembro). Os maiores valores de condutividade elétrica foram registrados para o reservatório Jacareí no segundo período estudado e os menores valores foram no reservatório Atibainha no primeiro período. Já as maiores concentrações de oxigênio dissolvido foram observadas nos meses de maio/ junho, principalmente na área de barragem do reservatório Atibainha, com 12,2 mg/L, fato que o caracterizou como saturado, caso típico de ambientes eutrofizados.

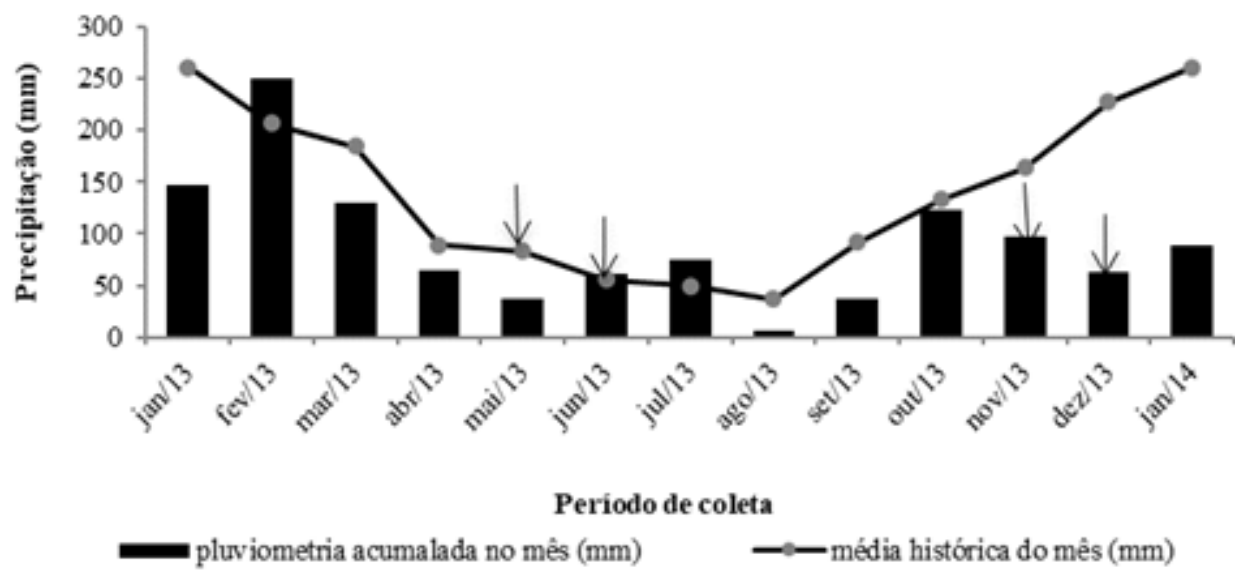

Fig. 2. Dados pluviométricos do Sistema Cantareira no ano de 2013. A seta indica os meses de coleta. 

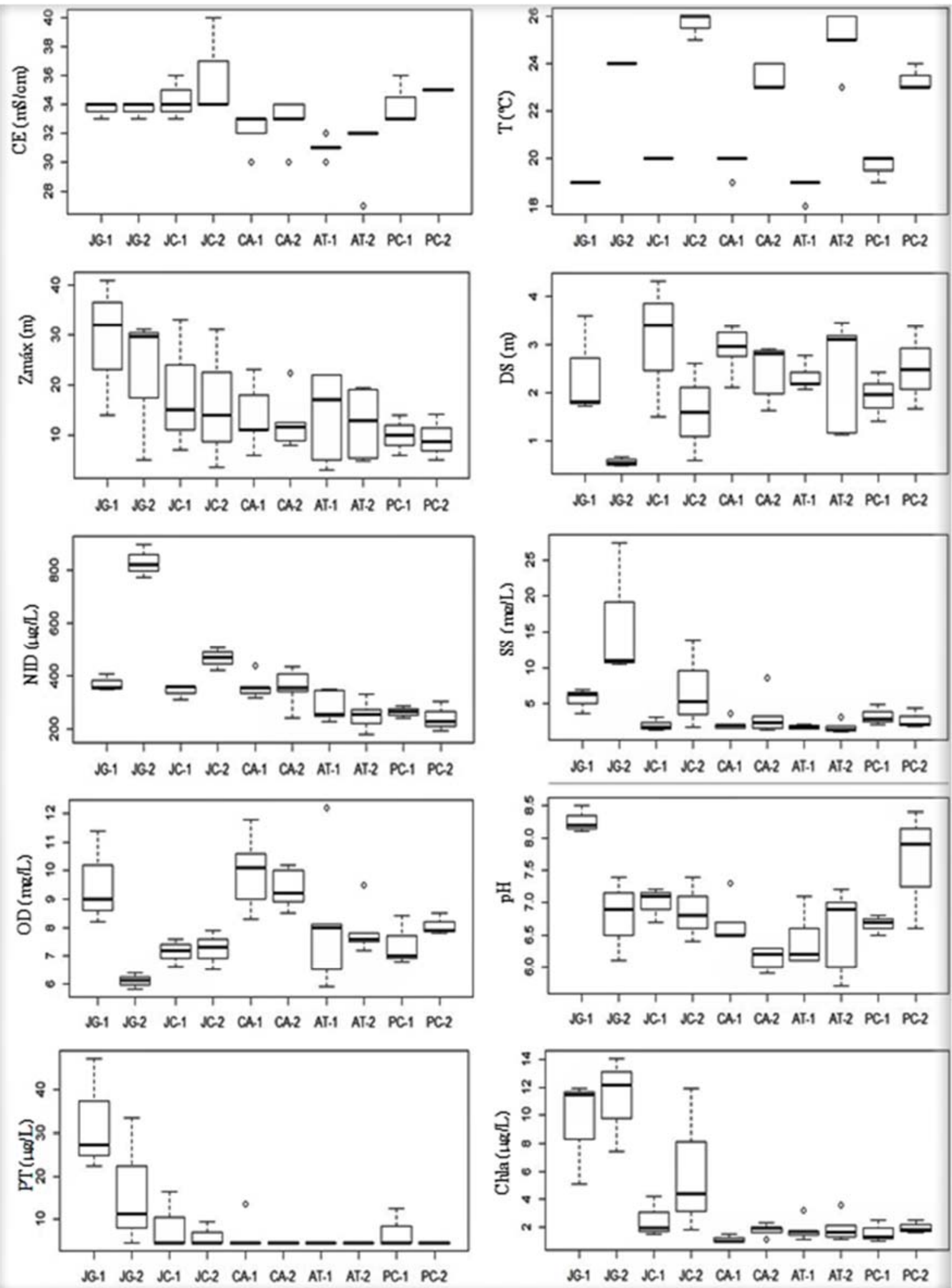

Fig. 3. Gráficos Box-Plot das variáveis ambientais analisadas nos períodos de maio/junho e novembro/dezembro de 2013, nos pontos de coletas dos reservatórios Jaguari-Jacareí, Cachoeira, Atibainha e Paiva Castro (Sistema Cantareira - SP). Condutividade elétrica (CE); temperatura (T); profundidade máxima (Zmáx); Disco de Secchi (DS); nitrogênio inorgânico dissolvido (NID); sólidos em suspensão (SS); oxigênio dissolvido (OD); potencial hidrogeniônico (pH); fósforo total (PT); clorofila- $a$ (Chla). Representação dos reservatórios nos meses de maio/junho: JG-1 (Jaguari); JC-1 (Jacareí); CA-1 (Cachoeira); AT-1 (Atibainha) e PC-1 (Paiva Castro) e novembro/dezembro: JG-2; JC-2; CA-2; AT-2 e PC-2. 
O índice de estado trófico entre os reservatórios variou de oligotrófico a supereutrófico. Para o reservatório Jaguari foram registrados os maiores graus de trofia, principalmente na área de transição para o reservatório Jacareí, os reservatórios Jacareí, Atibainha e Paiva Castro mantiveram-se mesotróficos. Já o reservatório Cachoeira nos períodos de coleta o índice de estado trófico variou de oligotrófico à mesotrófico, apresentando uma condição melhor em relação a qualidade da água quando comparado com os outros reservatórios (Fig. 4).

Esta variação nas trofias era relativamente esperada para o sistema, devido às características de uso e ocupação nas regiões próximas aos reservatórios, que podem influenciar o estado trófico no qual estarão enquadrados. Neste contexto, um estudo de caso realizado por Otomo et al. (2015), nas represas de Guarapiranga e Paiva Castro, durante todo o período analisado de 2006 a 2011, a represa de Guarapiranga foi classificada como eutrófica, já o reservatório Paiva Castro como mesotrófico na maior parte dos meses. Estas observações indicam a vulnerabilidade que os reservatórios próximos a grandes centros urbanos podem representar alterando suas trofias. Nesse caso, o Paiva Castro, embora tenha sido classificado pelos autores como mesotrófico, no presente estudo sua classificação ficou bastante próxima do limite do estado oligotrófico. Ainda assim, sua trofia permanece sujeita a fatores externos. Segundo Oliveira et al. (2008) o entorno da bacia hidrográfica dos reservatórios do Sistema Cantareira, observa-se que o uso e ocupação do solo se tornaram intensos e irregular com o passar do tempo, aumentando a poluição e contaminação das águas.

\section{Análise da comunidade fitoplanctônica}

Por meio da análise de correspondência canônica foi observada a formação de três grandes grupos dispostos em um gradiente horizontal, de modo que foi possível observar uma mudança na dinâmica e estrutura dos GFs fitoplanctônicos influenciadas pelas variáveis físicas, químicas e biológicas da água (Figs. 5 A, B).

De acordo com Cunha \& Calijuri (2011), a ocorrência de alguns grupos funcionais está diretamente relacionada ao estado trófico do sistema aquático e, nesse sentido, a abordagem fitossociológica pode oferecer indicativos do grau de enriquecimento do reservatório estudado.

Quanto à biomassa algal, as maiores contribuições foram quantificadas no reservatório Jaguari, nos dois períodos de coleta, sendo no período de maio/junho a maior contribuição foi do grupo dos dinoflagelados, principalmente pela espécie Ceratium furcoides, representada pelo grupo funcional Lo, que são compostos por organismos característicos de epilímnio de ecossistemas aquáticos mesotróficos no verão. Já no período de novembro/dezembro, a espécie predominante foi Cylindrospermopsis raciborskii do grupo das cianobactérias filamentosas, representado pelo grupo funcional $\mathrm{Sn}$, que são organismos competidores em ambientes túrbidos, podendo habitar ambientes eutróficos, estratificado ou misturado (Reynolds et al. 2002) (Quadro 1).

Nos dois períodos amostrais, foram observados 15 grupos funcionais fitoplanctônicos com abundância relativa acima de 5\% (A, B, C, E, F, Sn, J, K, Lo, M, N, P, X2, Y e W2) (Quadro 1).

De acordo com a ordenação das unidades amostrais, por meio da análise de correspondência canônica (ACC), com base na correlação de Pearson foram definidos onze grupos funcionais e oito variáveis ambientais no período de maio/junho, entretanto os eixos 1 e 2 não foram significativos $(p=0,30$ e $p=0,21)$, não ocorrendo correlação entre as variáveis ambientais e os grupos funcionais fitoplanctônicos. Os dois primeiros eixos da ACC explicaram 57,97\% da variação dos grupos funcionais em relação às variáveis ambientais (eixo $1=$ $33,37 \%$ e eixo $2=24,60 \%$ ) (Fig. $5 \mathrm{~A}$ ).

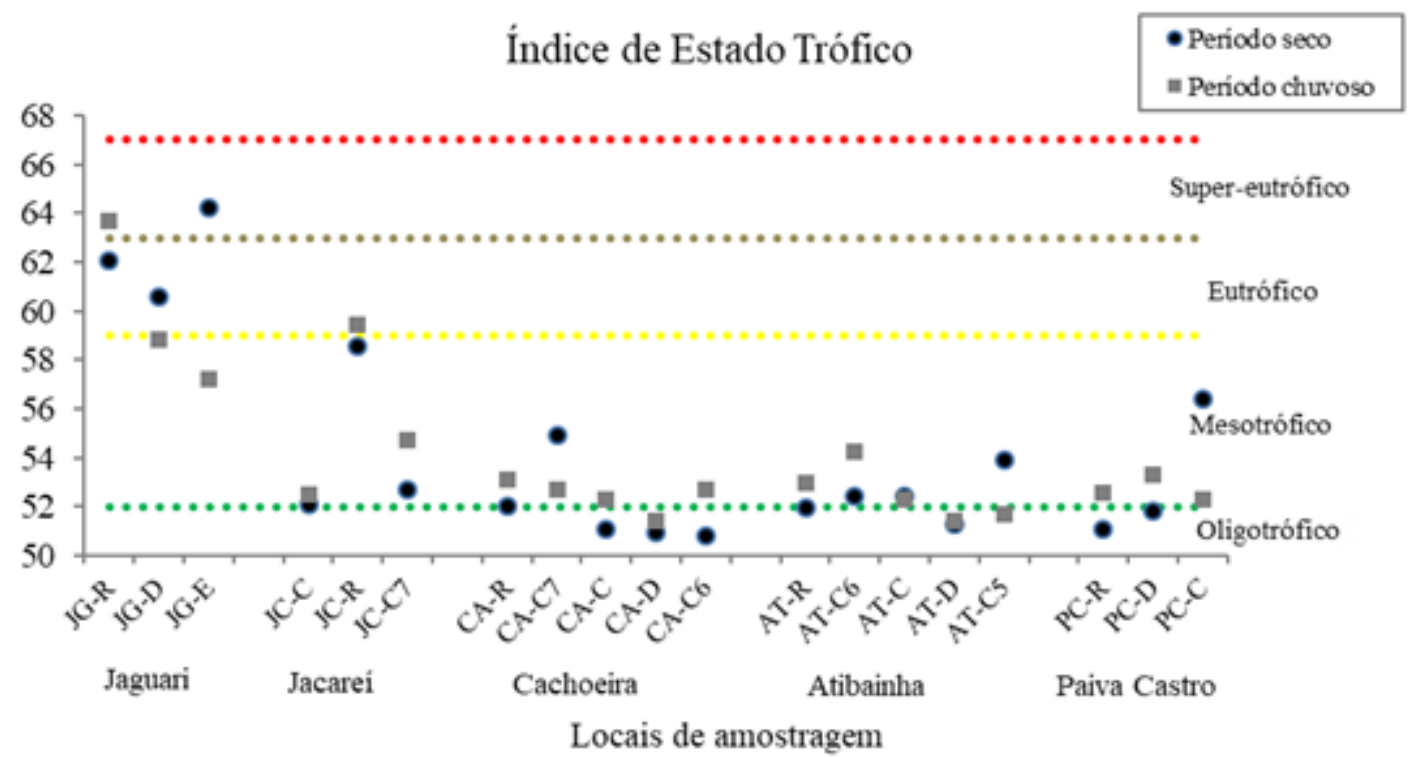

Fig. 4. Índice de Estado Trófico para os reservatórios do Sistema Cantareira, São Paulo. 
A

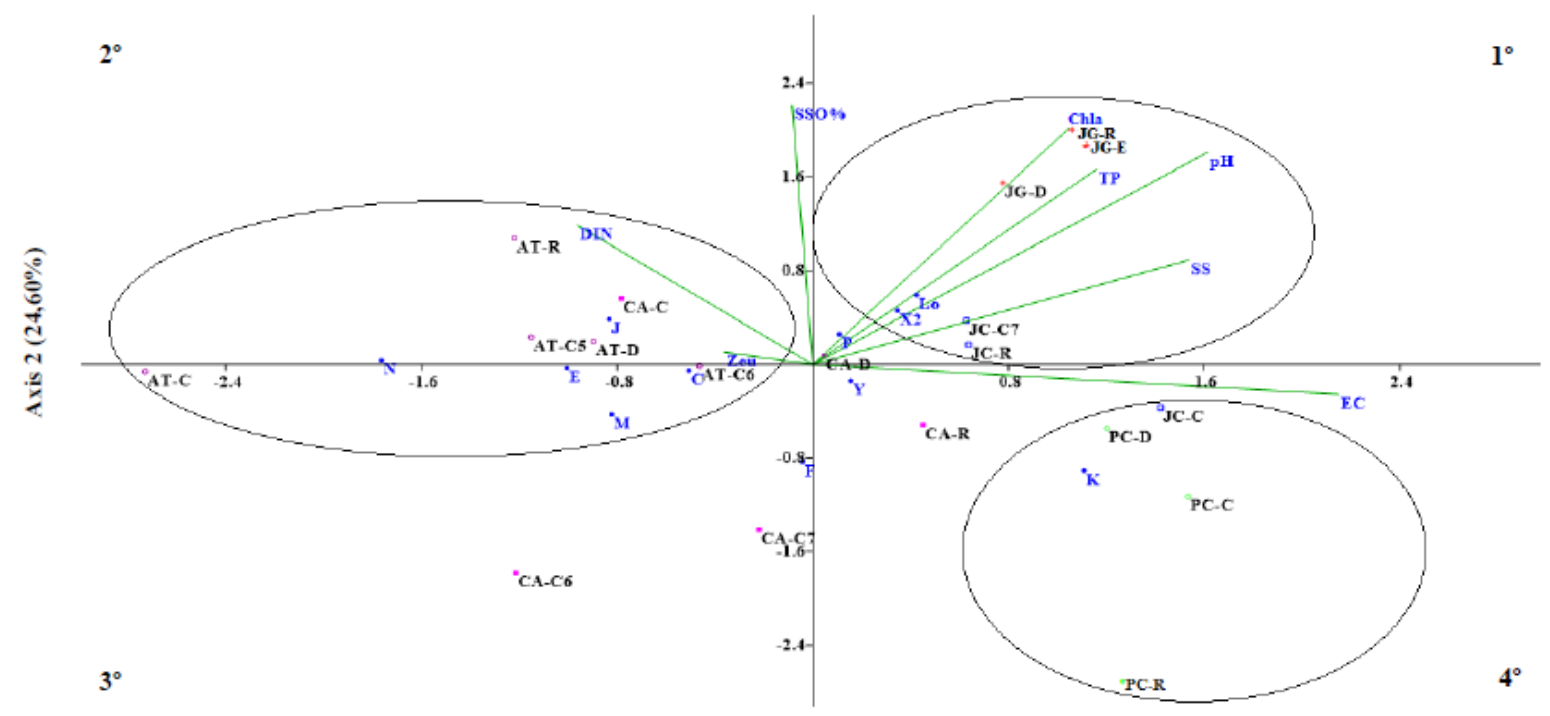

Axis $1(33,37 \%)$

B

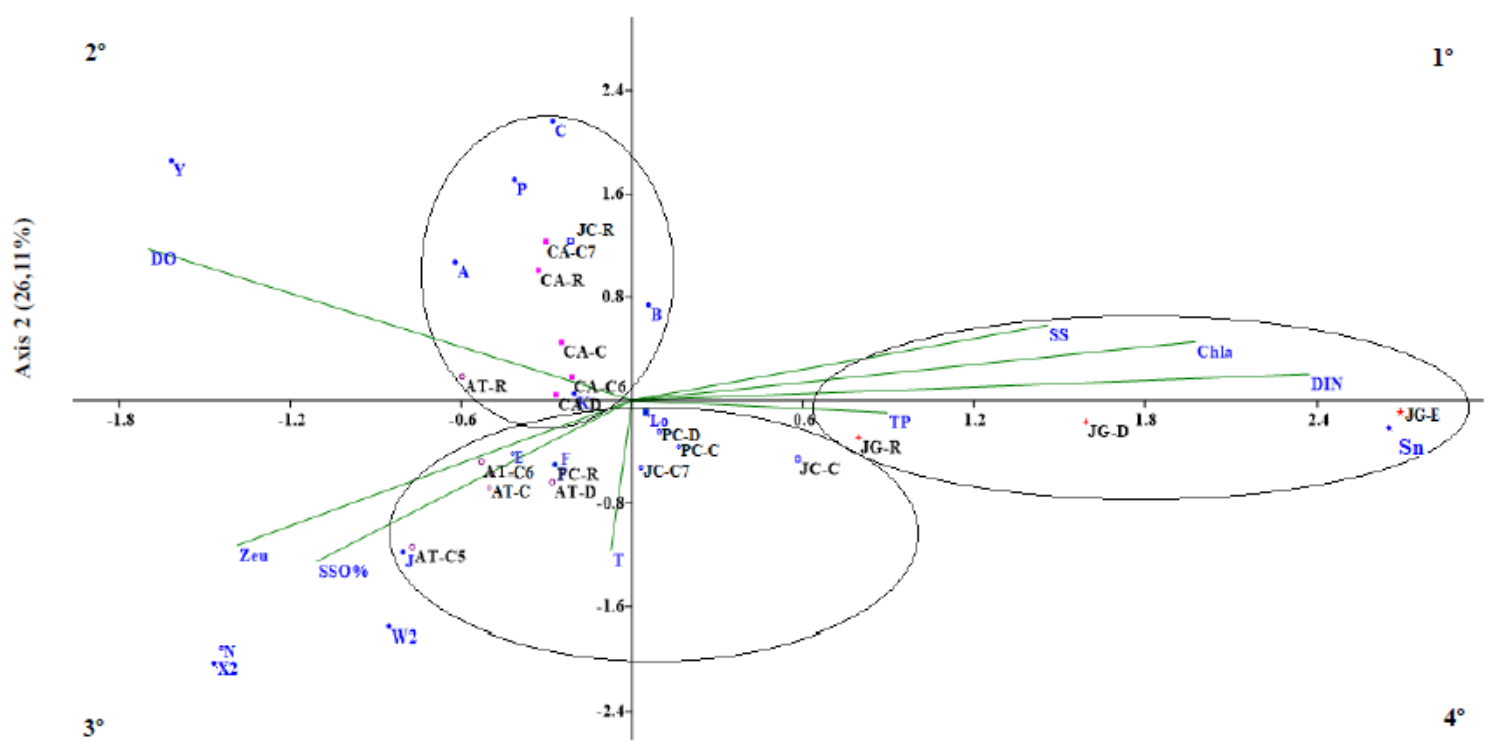

Figs. 5 A, B. Triplot da Análise de Correspondência Canônica para os grupos funcionais e as variáveis ambientais, nos 19 pontos de amostragem dos reservatórios do Sistema Cantareira, nos períodos de maio/junho (A) e novembro/dezembro (B) de 2013. Grupos Funcionais - A, B, C, E, F, J, K, Lo, M, N, P, Sn, X2, Y e W2). Variáveis Ambientais: pH (potencial hidrogeniônico); NID (nitrogênio inorgânico dissolvido); PT (fósforo total); Chla (clorofila-a); SS (sólidos em suspensão); CE (condutividade elétrica) e Zeu (zona eufótica); T (temperatura); \%SOS (fração de sólidos orgânicos) e OD (oxigênio dissolvido). Estações amostrais ao longo do eixo longitudinal (zonas fluvial, intermediária e lacustre): Reservatórios - Jaguari (JG-R, JG-D e JG-E); Jacareí (JC-C7, JC-R e JC-C); Cachoeira (CA-R, CA-C, CA-C6, CA-C7 e CA-D); Atibainha (AT-R, AT-C, AT-C5, AT-C6, AT-D) e Paiva Castro (PC-R, PC-C e PC-D). Período de estudo: Período seco (maio/junho) e período chuvoso (novembro/dezembro).

Já a ACC do período de novembro/dezembro, utilizou-se de quatorze grupos funcionais e oito variáveis ambientais, e o teste de Monte Carlo com permutação 999 apenas o eixo 1 foi significativo $(\mathrm{p}=0,01)$, o que indica que os acontecimentos não foram ao acaso, representando as semelhanças existentes entre as variáveis ambientais e os grupos funcionais fitoplanctônicos. Os eixos 1 e 2 obteve explicabilidade de $67,49 \%$ da variabilidade dos dados, sendo $41,38 \%$ pelo eixo 1 e $26,11 \%$ pelo eixo 2 (Fig. $5 \mathrm{~B}$ ).

Os pontos relativos ao período de novembro/dezembro tiveram uma ordenação mais agrupada em relação a maio/ junho, no qual os pontos ficaram ordenados distantes um do 
Quadro 1. Lista de grupos funcionais fitoplanctônicos de cada estação amostral com abundância relativa acima de 5\% e suas respectivas características de acordo com Reynolds et al. (2002), modificado por Brasil \& Huszar (2011).

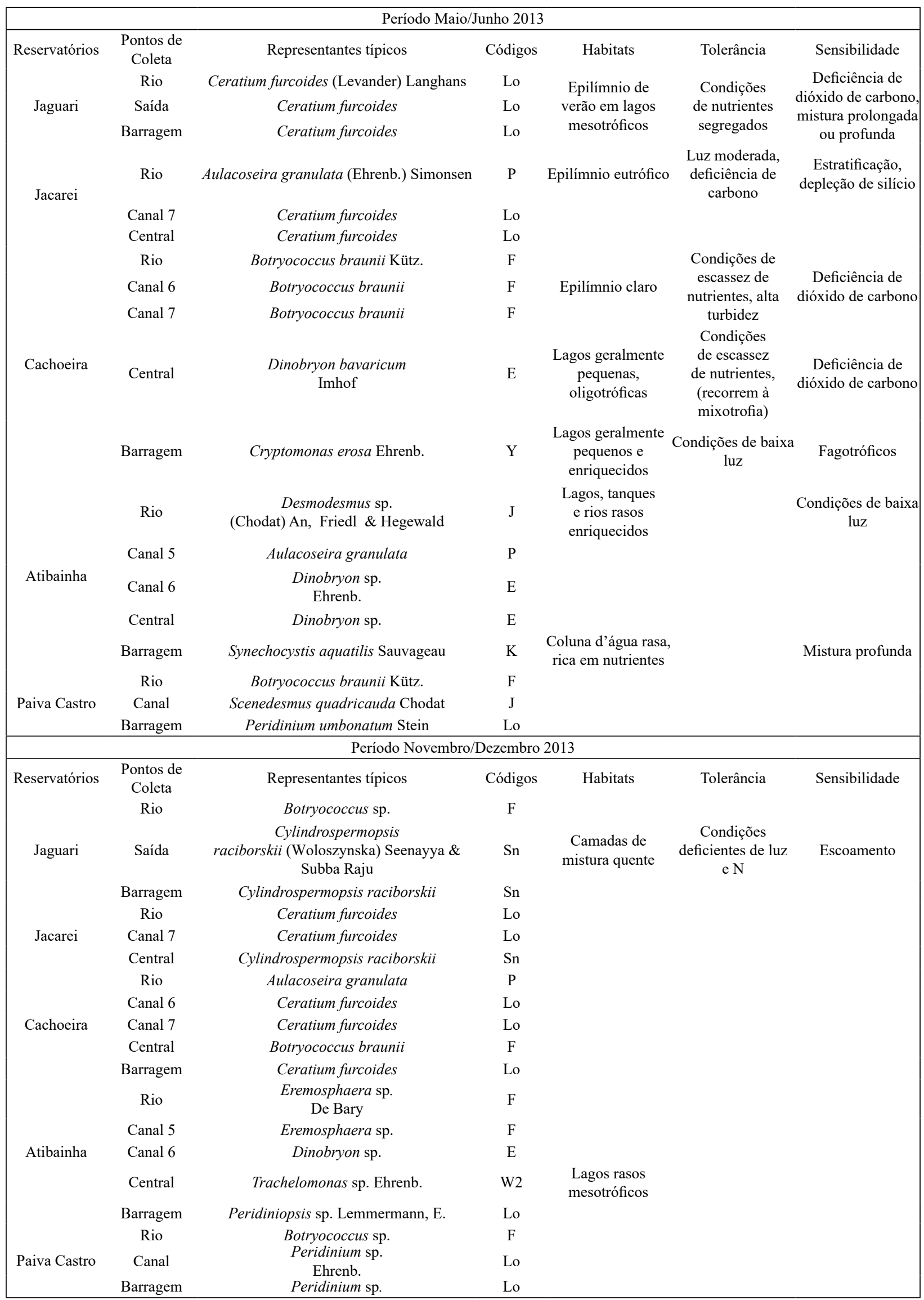


outro. Com isso, a análise indicou que, no segundo período, os GFs responderam de maneira mais específica à variação dos dados em cada local, ao passo que a ordenação das classes correlacionadas ao primeiro período foi mais homogênea.

$\mathrm{Na}$ figura $5 \mathrm{~A}$, os primeiros eixos da ACC explicaram a maior parte da correspondência entre os dados e ambas exibiram um padrão similar nos agrupamentos. Neste sentido, o reservatório Jaguari difere dos demais locais, em ambos os períodos sazonais, ficando ordenado às variáveis relacionadas à eutrofização (altas concentrações de PT, Chla, $\mathrm{pH}, \mathrm{SS}$ e NID) e a associação dessas variáveis com os GFs Lo (período maio/junho) e Sn (período novembro/ dezembro), os quais correspondem a ambientes eutrofizados e que evidenciou uma crescente degradação da qualidade da água. Resultados similares foram observados em outros estudos em reservatórios: Jurumirim (SP) (Henry et al. 1998) e Foz de Areia (PR) (Silva et al. 2005).

Entretanto, em direção aos reservatórios à jusante ocorreu uma melhora na qualidade da água, com destaque para os pontos centrais e barragens dos reservatórios Cachoeira e Atibainha, apresentando também maior diversidade de grupos funcionais $(\mathrm{K}, \mathrm{Y}, \mathrm{A}, \mathrm{P}, \mathrm{C}, \mathrm{B}, \mathrm{Lo}$, $\mathrm{F}, \mathrm{E}, \mathrm{J}, \mathrm{N}, \mathrm{W} 2 \mathrm{e} \mathrm{X} 2$ ). Tais grupos são tolerantes à baixa disponibilidade de luz a elevados valores de turbidez da água, além de representar determinados ambientes eutróficos e mesotróficos (Cunha \& Calijuri 2011).

Já os pontos de coleta dos reservatórios Cachoeira e Jacareí apresentaram-se dispersos nos dois períodos sazonais, dificultando realizar uma análise mais concreta dos dados. Contudo o reservatório Paiva Castro no primeiro período se diferenciou dos demais, com ocorrência do grupo funcional $\mathrm{K}$, comum em águas rasas e ricas em nutrientes, que por fim, caracterizou-se como um reservatório mesotrófico.

Os organismos fitoplanctônicos têm diferentes necessidades fisiológicas e respondem de modo distinto às variáveis físicas e químicas como luz, temperatura e regime de nutrientes (Miranda et al. 2013), corroborando o descrito neste trabalho.

No entanto, os grupos dominantes variam não apenas no espaço, mas também sazonalmente na medida em que as condições físicas, químicas e biológicas da água foram alteradas, como ocorreu com os grupos o período de maio/junho e Sn no período de novembro/dezembro, no reservatório a montante (Jaguari).

Quanto ao nível de trofia foi constatado que existe um gradiente longitudinal observado nas análises estatísticas (CCA), de modo que em cada reservatório foi possível observar uma diferença de nível trófico nos pontos onde foram realizadas as coletas, entretanto, a diferença foi mais visível na entrada do reservatório e na barragem.

Em síntese, foi constatada a existência da heterogeneidade espacial formando três grupos distintos no espaço amostral. O reservatório Jaguari, à montante, foi classificado com um elevado grau de trofia, porém, nos reservatórios Jacareí, Cachoeira e Atibainha concentrações de nutrientes relativamente menores foram identificadas, portanto, apresentando o menor valor no grau trófico, corroborando os resultados apresentados por De-Carli et al. (2017). Já no reservatório Paiva Castro, normalmente era de se esperar um aumento nessa trofia, devido às altas concentrações de nutrientes e material particulado em suspensão, contudo, não foram observadas grandes alterações se comparado ao reservatório Jaguari, possivelmente devido ao uso de algicida para controlar a proliferação de algas e plantas aquáticas.

Apesar disso, os reservatórios mais próximos de ambientes urbanos, indústrias e até mesmo de zonas rurais com práticas agropastoris, são ambientes que apresentam maiores trofias, constituindo principalmente os reservatórios, Jaguari e Paiva Castro.

Desta forma, por meio deste estudo foi possível verificar que os grupos funcionais respondem às variações de trofia nos reservatórios. Porém esta resposta é mais significativa em ambientes cujas concentrações das variáveis físicoquímicas são relativamente mais elevadas. Esta observação foi baseada segundo o que foi observado para o reservatório Jaguari, que se destaca dentre os demais com um alto grau trófico, e independente do período do ano, teve correlação com grupos funcionais característicos de ambientes eutrofizados. Quanto aos demais reservatórios, os grupos identificados são compatíveis aos de ambientes com um grau trófico menor em relação ao Jaguari, concomitantemente, as características físico-químicas corresponderam a este resultado.

Estas observações demonstram que o uso dos grupos funcionais como ferramenta de monitoramento ambiental é eficaz na sintetização das informações sobre a dinâmica e estrutura dos grupos fitoplanctônicos. Entretanto, sua aplicação deve, necessariamente, ser utilizada em uma análise conjunta com as variáveis ambientais visando uma abordagem mais representativa dos ecossistemas aquáticos.

\section{REFERÊNCIAS}

Anagnostidis, K. \& Komárek J. 1988. Modern approach to the classification system of Cyanobacteria. 3- Oscillatoriales. Archiv für Hydrobiologie. Supplement Algological Studies. 80(1-4):327-472.

1990. Modern approach to the classification system of Cyanobacteria. 5-Stigonematales. Archiv für Hydrobiologie. Supplement Algological Studies 59(1):73.

Barros, P.P.S. 2012. Análise de diferentes cenários para adequação ambiental da Microbacia Hidrográfica do Ceveiro. Dissertação 126 f., Escola Superior de Agricultura Luiz de Queiroz, Universidade de São Paulo, Piracicaba, São Paulo.

Becker, V., Caputo, L., Ordóñez, J., Marcé, R., Armengol, J., Crossetti, L.O. \& Huszar V.L.M. 2010. Driving factors of the phytoplankton functional groups in a deep Mediterranean reservoir. Water Research 44(11):3345-3354. https://doi.org/10.1016/j.watres.2010.03.018

Bicudo, C. E. M. \& Menezes, M. 2006. Gêneros de algas de águas continentais do Brasil: Chave para identificação e descrições. Editora Rima, São Paulo. 502 p.

Brasil, J. \& Huszar, V.L.M. 2011. O papel dos traços funcionais na ecologia do fitoplâncton continental. Oecologia Australis 15(4):799834. http://dx.doi.org/10.4257/oeco.2011.1504.04

Buzelli, G.M. \& Cunha-Santino, M.B. 2013. Análise e diagnóstico da qualidade da água e estado trófico do reservatório de Barra Bonita (SP). Revista Ambiente \& Água 8(1):186-205. http://dx.doi. org/10.4136/ambi-agua.930 
Cardoso-Silva, S., Nishimura, P.Y., Padial, P.R., Mariani, C.F., MoschiniCarlos \& Pompêo, V.M.L.M. 2014. Compartimentalização e qualidade da água: o caso da Represa Billings. Revista Bioikos 28(1):31-43. Disponível em: $<$ http://periodicos.puc-campinas.edu.br/seer/index. php/bioikos/article/view/2522/1864> Acessado em 15.03.2017.

Carlson, R.E. 1977. A trophic state index for lakes. Association for the Sciences of Limnology and Oceanography 22(2): 361-369. https:// doi.org/10.4319/lo.1977.22.2.0361

Companhia de Saneamento Básico do Estado de São Paulo - SABESP. 2014. Mananciais da região metropolitana de São Paulo - O Sistema Cantareira. Disponível em: http://www.mananciais.org.br/site/ mananciais rmsp/cantareira Acessado em: 03.04.2016.

Cunha, D.G.F. \& Calijuri, M.C. 2011. Variação sazonal dos grupos funcionais fitoplanctônicos em braços de um reservatório tropical de usos múltiplos no estado de São Paulo (Brasil). Acta botânica brasílica 25(4):822-831. http://dx.doi.org/10.1590/S0102-33062011000400009

De-Carli, B.P., Lopez-Dorval, J.C., Rodrigues, E.H.C. \& Pômpeo, M.L.M. 2017. Variação espacial e sazonal do zooplâncton nos reservatórios do Sistema Cantareira, Brasil. Revista Ambiente \& Água 12(4):666679. http://dx.doi.org/10.4136/ambi-agua. 1935

Giatti, L.L. 2000. Reservatório Paiva Castro - Mairiporã- SP: avaliação da qualidade da água sobre alguns parâmetros físicos, químicos e biológicos (1987-1998). Dissertação 87 p., Faculdade de Saúde Pública da Universidade de São Paulo, São Paulo.

Hammer, Ø., Harper, D.A.T. \& Ryan, P.D. 2001. PAST: paleontological statistics software package for education and data analysis. Palaeontologia Electronica. 4(1):9. Disponível em: < https:// palaeo-electronica.org/2001 1/past/issue1 01.htm $>$ Acessado em 26.09.2016.

Henry, R., Nunes, M.A., Mitsuka, P.M., Lima, N. \& Casanova, S.M.C. 1998. Variação espacial e temporal da produtividade primária pelo fitoplâncton na represa de Jurumirim (rio Paranapanema, SP). Revista Brasileira de Biologia 58(4):571-590. http://dx.doi.org/10.1590/ S0034-71081998000400005

Hillebrand, H., Durselen, C.D., Kirscheterl, D., Pollingher, U. \& Zohary, T. 1999. Biovolume calculation for pelagic and benthic microalgae. Journal of Phycology 35(2):403-424. https://doi.org/10.1046/j.15298817.1999.3520403.x

Instituto da Água - INAG IP. 2009. Manual para a avaliação da qualidade biológica da água. Protocolo de amostragem e análise para o Fitoplâncton Ministério do Ambiente, do Ordenamento do Território e do Desenvolvimento Regional. Instituto da Água I.P., Lisboa, 67 p.

Komárek, J. \& Anagnostidis, K. 1986. Modern approach to the classification system of Cyanophytes, 2: Chroococcales. Archiv für Hydrobiologie / Algological Studies (43):157-226.

2005. Cyanoprokayota 2 Teil: Oscillatoriales. In: Bridel, B., Gaster, G., Krienitz, L. \& Schargerl, M. (Eds.). Subwasserflora von Mitteleuropa. Müncher: Elsevier. 759 p.

Koroleff, F. 1976. Determination of nutrients. In Methods of sea water analysis (K. (Grasshoff, ed.) Verlag Chemie Weinheim, New York, $117-181 \mathrm{p}$

Krammer, K. \& Lange-Bertalot H. 1986. Bacillariophyceae. 1. Teil: Naviculaceae. In Süßwasserflora von Mitteleuropa (H. Ettl et al., eds). Gustav Fisher Verlag, Stuttgart, 2(1):876.

Lamparelli, M.C. 2004. Grau de trofia em corpos d'água do estado de São Paulo: Avaliação dos métodos de monitoramento. Tese $238 \mathrm{f}$, Instituto de Biociências, Universidade de São Paulo, São Paulo.

Lorenzen, C.J. 1967. Determination of chlorophyll and pheo-pigments: Spectrophotometric equations. Association for the Sciences of Limnology and Oceanography 12(2):343-346. http://dx.doi. org/10.4319/lo.1967.12.2.0343

Lund, J.W.G., Kipling, C. \& Lecren, E.O. 1958. The inverted microscope method of estimating algal number sand the statistical basis of estimations by counting. Hydrobiologia 11(2):143-170.

Mackereth, F.J.H., Heron, J. \& Talling, J.F. 1978. Water analysis: Some revised methods for limnologists. Freshwater Biological Association Scientific Publication. Titus Wilson \& Son Ltd, Kendall., (36):120.
Miranda, C.S, Alexandre, G.S. \& Negreiros, N.F. 2013. Caracterização da comunidade fitoplanctônica do reservatório de abastecimento público e estação de tratamento de água no município de GuararapesSP, Brasil. Periódico eletrônico Fórum Ambiental da Alta Paulista 9(11):418-430. http://dx.doi.org/10.17271/198008279112013687

Moraes, J.M., Pellegrino, G.Q., Ballester, M.V., Martinelli, L.A., Victoria, R.L. \& Krusche, A.V. 1998. Trends in Hydrological Parameters of a Southern Brazilian Watershed and its Relation to Human Induced Changes. Water Resources Management 12(4):295-311.

Nascimento, M.N. 2012. Biodiversidade e distribuição das diatomáceas planctônicas e de sedimento superficial em represa profunda oligotrófica (Sistema Cantareira, Represa Jaguari-Jacareí). São Paulo. Dissertação 151 p., Instituto de Botânica da Secretaria de Estado do Meio Ambiente, São Paulo.

Navarro, E., Bacardit, M., Caputo, L., Palau, T. \& Armengol, J., 2006. Limnological characterization and flow patterns of a three-coupled reservoir system and their influence on Dreissena polymorpha populations and settlement during the stratification period. Lake and Reservoir Management 22(4):293-302. https://doi. org/10.1080/07438140609354363

Nishimura, P.Y. 2008. Ecologia da comunidade fitoplactônica em dois braços da Represa Billings (São Paulo, SP) com diferentes graus de trofia. Dissertação 148 p., Instituto de Biociência, Universidade de São Paulo, São Paulo.

Nishimura, P.Y., Moschini-Carlos, V. \& Pompêo, M. 2015. O estudo fitoplâncton com base nos grupos funcionais: origens e um vislumbre sobre seu futuro. In (Marcelo Pompêo Org.). Grupos funcionais do fitoplâncton. Ecologia de reservatórios e interfaces. São Paulo: Universidade de São Paulo, v. 1, part. 1, p. 120-130.

Oliveira, P.E., Gomes, A.R., Sanches, R.C. \& Saad, A.R. 2008. Análise da evolução da paisagem no entorno da represa dos rios Jaguari e Jacareí, Estado de São Paulo, com base em sensoriamento remoto e SIG. Revista Geociência 27(4):527-539.

Otomo, J.I., Cardoso-Silva, S., Santos, W.D.S., Jardim, E.A. M. \& Pômpeo, M. 2015. Avaliação de políticas para preservação e recuperação de mananciais de abastecimento público da Região Metropolitana de São Paulo. In. Avaliação de política pública em áreas de mananciais. Ecologia de reservatórios e interfaces. (Marcelo Pompêo Org.). Universidade de São Paulo, São Paulo, v. 1, part. 1, p. 376-395.

Padisák, J., Crossetti, L.O. \& Naselli-Flores, L. 2009. Use and misuse in the application of the phytoplankton functional classification: a critical review with updates. Hydrobiologia 621(1):1-19. https://10.1007/ s10750-008-9645-0

Pinto-Coelho, R.M., Coelho, M.M., Espírito-Santo, M.M. \& Cornelissen, T.G. 1999. Efeitos da eutrofização na estrutura da comunidade planctônica na lagoa da Pampulha. In: Henry, R. ed. Ecologia de reservatórios: estrutura, função e aspectos sociais. (R. Henry, ed.), Fundação do Instituto de Biociências, Botucatu, p. 551-572.

Pompêo, M., Moschini-Carlos, V., López-Doval, J.C., Abdalla-Martins, N., Cardoso-Silva, S., Freire, R.H.F, Beghelli, F.G.S, Brandimarte, A.L., Rosa, A.H. \& López, P. 2017. Nitrogen and phosphorus in cascade multi-system tropical reservoirs: water and sediment. Limnological Review 17(3):133-150. https://doi.org/10.1515/ limre-2017-0013

Reynolds, C., Huszar, V.L.M., Kruk, C., Naselli-Flores, L. \& Melo, S. 2002. Towards a functional classification of the freshwater phytoplankton. Journal of Plankton Research 24(5):417-428. https:// doi.org/10.1093/plankt/24.5.417

Round, F.E. 1965. The Biology of the Algae. London: Edward Arnold (Publishers); Ltd. Journal of the Marine Biological Association of the United Kingdom, v. 45, 269 p. https//:10.1017/S0025315400016672 1971. The Taxonomy of the Chlorophyta, II. British Phycological Journal 6(2):235-264.

Santos, R.M. 2014. Estruturas das comunidades fito e zooplanctônicas do Reservatório Guarapiranga (São Paulo) e relações com a hidrodinâmica e a eutrofização. Tese 239f., Universidade de São Carlos, São Carlos-SP. 
Serafim-Júnior, M., Perbiche-Neves, G., Brito, L., Ghidini, A.R. \& Casanova, S.M.C. 2010. Variação espaço-temporal de rotífera em um reservatório eutrofizado no sul do Brasil. Iheringia Série Zoologia 100(3):233-241. https://doi.org/10.1590/S007347212010000300008

Silva, C.A., Train, S. \& Rodrigues, C.L. 2005. Phytoplankton assemblages in a Brazilian subtropical cascading reservoir system. Hydrobiologia 537(1-3):99-109. https://doi.org/10.1007/s10750-004-2552-0

Sun, J. \& Liu, D. 2003. Geometric models for calculating cell biovolume and surface area for phytoplankton. Journal of Plankton Research 25(11):1331-1346. https://doi.org/10.1093/plankt/fbg096

Tavares, T.R., Martello, P.R.F.M., Monteiro, D. \& Strabeli, T.F. 2015. Uso de geotecnologias para monitoramento da vegetação florestal nativa em períodos de seca no município de Piracicaba-SP. In Anais do XLIV Congresso Brasileiro de Engenharia Agrícola, São Pedro, São Paulo,

Ter Braak, C.J.F. 1986. Canonical Correspondence Analysis: A new eigenvector technique for multivariate analysis. Ecology, v. 67, 1167-1179 p. http://dx.doi.org/10.2307/1938672
Torgan, L.C. 1997. Estrutura e dinâmica da comunidade fitoplanctônica na laguna dos Patos, Rio Grande do Sul, Brasil, em um ciclo anual. Tese 284 f., Universidade Federal de São Carlos, São Carlos.

Torgan, L.C., Becker, V. \& Prates, H.M. 1999. Checklist das diatomáceas (Bacillariophyceae) de ambientes de águas continentais e costeiros do estado do Rio Grande do Sul. Iheringia. Série Botânica 52:89-144.

Uehlinger, V. 1964. Étude statistique des méthodes de dénombremente planctonique. Archives des Sciences Physiques et Naturelles 17(2):121-223.

Utermohl, H. 1958. Zur Vervollkommnung der quantitativen phytoplankton-methodik. Mitteilungen Internationalen Vereinigung für theoretische und angewandte Limnologie 19:1-38.

Valderrama, J.C. 1981. The simultaneous analysis of total nitrogen and phosphorus in natural waters. Marine Chemistry 10(2):109-122. https://doi.org/10.1016/0304-4203(81)90027-X

Wetzel, R.G. \& Likens, G.E. 1991. Limnological Analyses. SpringerVerlag, New York. 391 p.

Whately, M. \& Cunha, P. 2007. Cantareira 2006: um olhar sobre o maior manancial de água da Região Metropolitana de São Paulo (Ong. Instituto Socioambiental). São Paulo. 68 p. 